\title{
Medizinische Betreuung à la française
}

\author{
Ueli Bollag \\ Dr. med., Facharzt für Kinder und Jugendmedizin
}

Hurra, geschafft, den Mont Ventoux mit meinem Renner erreicht. Doch zu welchem Preis. Oben angekommen, erschöpft, vermochte ich mich nicht aus den Pedalen auszuklinken, stürzte nach links, direkt auf die Hüfte und Knack, mir war klar, Femurfraktur. Hier beginnt meine Erzählung.

Unfähig, mich zu rühren, lag ich da, von vielen Gaffern umrundet. Die angeforderte Ambulanz liess auf sich warten; mir war kalt und ich hatte Schmerzen. Eine Stunde später kam der Wagen dann angebraust, doch nicht ausgerüstet für den Transport eines Verletzten. Ich konnte schliesslich in die zweite Ambulanz - weitere 30 Minuten waren vergangen und ich lag noch stets verkrümmt auf der Strasse - gehievt werden. Einmal im Spital von Carpentras angekommen, wurde ich auf eine interne Bahre umgelagert und beim Perron 8 deponiert. Da lag ich wieder, noch stets in meiner verschwitzten Velofahrer Montur, durstig, müde. Nach einer weiteren Wartezeit von einer Stunde pochte ich dann darauf, dass nun endlich ein Röntgenbild erstellt würde. Den Auftrag dazu gab's dann von einem "Confrère», wohl dank dessen, dass ich mich als Arzt zu erkennen gegeben hatte. Halt vor der geschlossenen radiologischen Station - Samstagnachmittag, niemand zur Stelle und somit zurück auf Feld 8 - es lebe die 35-Stunden Woche in Frankreich. Das gewünschte Röntgenbild konnte dann nach weiteren 30 Minuten doch noch angefertigt werden. Wie erwartet, eine pertrochantere Femurfraktur links. Wie weiter? Jedenfalls nicht hier nach all den fragwürdigen Service Leistungen, die mein Zutrauen zu dieser Klinik schwinden liessen. Stattdessen - meine Frau war mir im eigenen Auto gefolgt und übernahm die Erledigung des bereits anschaulichen Papierkrams weiter nach Béziers in die Nähe unseres Zweitheims in Frankreich. Zwischenzeitlich war es 19:00 abends, und noch nie hatte mich jemand gefragt, ob ich zu trinken wünsche oder ob man mich waschen und umkleiden könnte. Nach einer weiteren Stunde teilte mir der diensttuende Arzt mit, dass sie nicht über das notwendige Werkzeug verfügten, eine pertrochantere Femurfraktur zu stabiliseren. Also Verfrachtung ins nahegelegene Spital von Béziers. Erstmals traf ich dort auf eine kompetente Pflegeequipe. Ins Zimmer kutschiert, er- hielt ich nun zu vorgerückter Stunde zu essen und zu trinken. Natürlich fand ich keinen Schlaf. Immer wieder musste ich das Unfallgeschehen rekonstruieren und das Versagen, mich aus den Pedalen zu befreien, beklagen. Von nun an blieb ich "à jeun" - ich stand auf der sonntäglichen Liste der zu operierenden Patienten. Der Eingriff fand dann genau 18 Stunden später statt. Keinerlei Infusion, um die zweite Periode von Deshydratation zu vermeiden. Ich greife vor: während der ersten Nacht zu Hause musste ich auch noch eine Nierenkolik durchstehen, nicht weiter erstaunlich, oder?

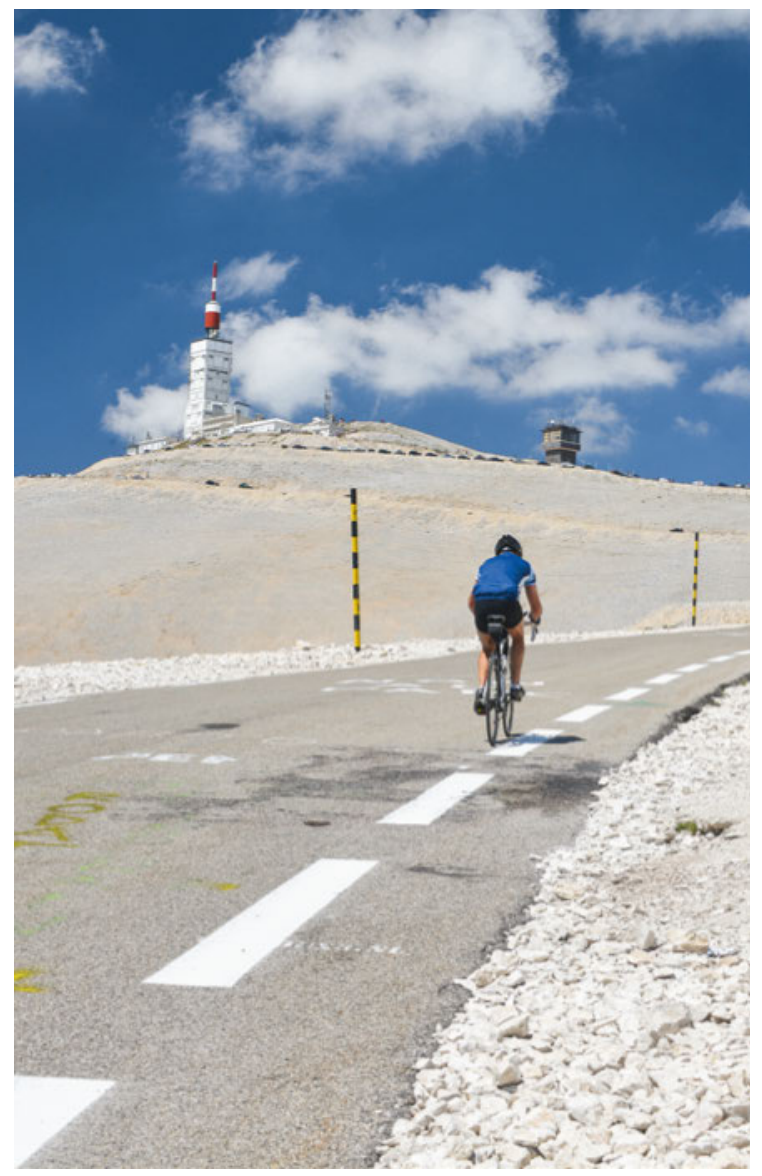

Jedes Jahr machen sich unzählige Menschen per Bike auf zum Mont Ventoux. Auch unser Autor hat die Spitze in 1912 Metern Höhe erreicht - doch beim Absteigen zog er sich eine Femurfraktur zu, sodass er sich unversehens in der Rolle des Patienten wiederfand (Symbolbild). 
Das operative Geschehen verlief reibungslos, sowohl was den chirurgischen Eingriff wie auch die Anaesthesie betrifft. Zurück im Zimmer, gab's dann noch etwas lauwarmes Hahnenwasser und etwas zu essen, für mich ungeniessbar. Immerhin tropfte nun eine Infusion, mit einem Schmerzmittel versehen, in meinen rechten Arm. So gegen Morgen, $07 \mathrm{Uhr}$, wurde mir der Blutdruck und die Temperatur gemessen, Routine eben, kaum ein Wort mit dem Patienten. Zum Morgenessen Brot, Butter, Konfitüre und eine Tasse Milchkaffee (keine zweite). Ich klingle, denn die Infusion läuft nicht mehr bzw. hat die Talsohle erreicht. Antwort: keine, und so deinstalliere ich das Zeugs eben selbst. Die Schmerzen sind nicht eigentlich mein Problem, doch hier wird allen Patienten, ob notwendig oder nicht, die Allerweltskapsel Dolipran (Paracetamol) $3 \times 2$ à 500 mg serviert, welcher Art die Schmerzen auch immer! Schon wieder bleiben Urinreste im Urinal, hier als «pistolet» bekannt, und Reste vom vorabendlichen Zähneputzen auf dem Tisch liegen, auf dem auch das Essen serviert wird, recht unappetitlich.

Hier sind verschiedene Betreuungsequipen am Werk, die eine fürs Essen, die andere für die Hygiene, und noch eine andere für die medizinischen Routinekontrollen verantwortlich, doch die Verbindung zwischen diesen Gruppen fehlt eben. Schichtwechsel, in eine solche Zeit fiel einmal die Betätigung meiner Klingel. Ich müsse wissen, dass die Ablöse noch eine weitere halbe Stunde dauern werde - keine Hilfe während dieser Zeit, die Schichtwechselprozedur hat Vorrang vor den Wünschen des Patienten. Die Hilfe kam dann doch noch, aber nicht 30 Minuten, sondern 90 Minuten später!

Fehlende Pflege und nur sporadisches Eingehen auf den Patienten; ich hielt das nicht mehr aus, wurde trübselig. Nur eineinhalb Tage nach der Operation wollte ich dieses Haus verlassen. Glücklicherweise konnte ich den «Kine» (Physiotherapeuten) davon überzeugen, dass ich mit Krücken umzugehen weiss, und auch der exzellente Chirurge traute mir zu, zu Hause zurechtzukommen.

\section{Diskussion}

- Das schweizerische Gesundheitssystem liegt gemäss Euro Health Consumer Index (EHCI) auf dem zweiten Platz, hinter Holland und vor Norwegen. Die schlechte Nachricht: Beim Preis-Leistungsverhältnis belegt die Eidgenossenschaft lediglich Rang 19, zwei Ränge hinter Frankreich.

- Wie meine Schilderung zeigt, müssen solche auf messbaren Parametern beruhenden Analysen mit Vorsicht betrachtet werden. So können zwar technische Errungenschaften in Zahlen gemessen werden.
Da bestehen kaum grosse Unterschiede zwischen der Schweiz und Frankreich. Der pflegerische Bereich allerdings, ein teurer Posten, lässt sich nur mit qualitativen Methoden untersuchen, eben etwa so, wie in meinem Fall geschehen. Und da öffnen sich gewaltige Gräben zwischen den zwei Ländern.

- Jetzt sieht die Rechnung noch einmal anders aus. Bei ungefähr gleichen Ausgaben fürs Gesundheitswesen in Frankreich und der Schweiz, erweist sich Frankreich im qualitativen Bereich der Schweiz deutlich unterlegen. Ich wage, diese Aussage zu machen, weil ich schon mehrmals in schweizerischen Spitälern wegen eines Unfalls zu meiner höchsten $\mathrm{Zu}$ friedenheit betreut worden bin, jedenfalls viel besser als hier in Frankreich. Oder finden sie, dass es sich hier um einen Einzelfall handle und man nicht generalisieren könne? Dann fragen sie französische Staatsangehörige, so wie ich es immer wieder tue, und da wird landauf, landab von ähnlichen Missständen in den Spitälern gesprochen.

\section{Quintessenz}

Das schweizerische Gesundheitssystem ist teuer und schneidet im internationalen Vergleich gemäss EHCI nur mittelmässig ab, wenn man das Preis-Leistungsverhältnis zum Vergleich heranzieht. Ginge man aber auf qualitative Werte ein, wie es eben der Pflegebereich darstellt, käme die Schweiz wieder besser weg, sicher besser als Frankreich.

\section{Aufruf}

Wenn uns die Gesundheit viel bedeutet, und das tut sie ja, stehen wir doch zu unserem teuren Gesundheitswesen. Schätzen wir den hohen Standard im Pflegebereich und tragen wir dazu bei, dass dies so bleibe, auch wenn es etwas kostet.

Bildnachweis

(c) Xxlphoto|Dreamstime.com

\section{Neue Serie \\ «Ärztinnen und Ärzte als Patienten»}

Haben Sie sich als Ärztin oder Arzt auch schon unversehens in der Patientenrolle wiedergefunden? Wie haben Sie diesen Perspektivenwechsel erlebt? Wenn Sie finden, dass Ihre Erfahrungen für die Leserschaft der SÄZ interessant sein könnten, möchten wir Sie dazu animieren, uns dazu einen Artikel von maximal 8000 Zeichen Umfang an redaktion.saez[at]emh.ch einzureichen. Ihr Manuskript wird dann von der Redaktion geprüft und bei positiver Beurteilung im Rahmen der Serie «Ärztinnen und Ärzte als Patienten" publiziert. 